\title{
Antibodies to SARS-CoV-2 protect against re-infection during outbreaks in care homes, September and October 2020
}

Anna Jeffery-Smith ${ }^{1}$, Nalini lyanger ${ }^{2}$, Sarah V Williams ${ }^{2}$, J Yimmy Chow ${ }^{2}$, Felicity Aiano ${ }^{1}$, Katja Hoschler ${ }^{1}$, Angie Lackenby ${ }^{1}$, Joanna Ellis ${ }^{1}$, Steven Platt ${ }^{1}$, Shahjahan Miah ${ }^{1}$, Kevin Brown ${ }^{1}$, Gayatri Amirthalingam ${ }^{1}$, Monika Patel ${ }^{1}$, Mary E Ramsay $^{1}$, Robin Gopal $^{1}$, Andre Charlett ${ }^{3}$, Shamez N Ladhani ${ }^{1}$, Maria Zambon ${ }^{1}$

1. National Infection Service Public Health England, London, United Kingdom

2. London Coronavirus Response Centre, Public Health England, London, United Kingdom

3. Data and Analytical Sciences, Public Health England, London, United Kingdom

Correspondence: Anna Jeffery-Smith (anna.jefferysmith@phe.gov.uk)

Citation style for this article:

Jeffery-Smith Anna, Iyanger Nalini, Williams Sarah V, Chow J Yimmy, Aiano Felicity, Hoschler Katja, Lackenby Angie, Ellis Joanna, Platt Steven, Miah Shahjahan, Brown Kevin, Amirthalingam Gayatri, Patel Monika, Ramsay Mary E, Gopal Robin, Charlett Andre, Ladhani Shamez N, Zambon Maria. Antibodies to SARS-CoV-2 protect against re-infection during outbreaks in care homes, September and October 2020. Euro Surveill. 2021;26(5):pii=2100092. https://doi.org/10.2807/15607917.ES.2021.26.5.2100092

Two London care homes experienced a second COVID19 outbreak, with 29/209 (13.9\%) SARS-CoV-2 RT-PCRpositive cases (16/103 residents, $13 / 106$ staff). In those with prior SARS-CoV-2 exposure, 1/88 (1.1\%) individuals (antibody positive: 87 ; RT-PCR-positive: 1) became PCR-positive compared with $22 / 73$ (30.1\%) with confirmed seronegative status. After four months protection offered by prior infection against re-infection was $96.2 \%$ (95\% confidence interval (CI): 72.7-99.5\%) using risk ratios from comparison of proportions and 96.1\% (95\% Cl: 78.8-99.3\%) using a penalised logistic regression model.

In autumn 2020, two care homes in London, United Kingdom (UK) with high rates of severe acute respiratory syndrome coronavirus 2 (SARS-CoV-2) seropositivity following outbreaks in the first wave of the coronavirus disease (COVID-19) pandemic [1,2] experienced a second COVID-19 outbreak. Outbreak investigations and SARS-CoV-2 serology were repeated to assess the role of antibodies in protecting against SARS-CoV-2 re-infection.

\section{Affected care homes \\ Care home A provides residential and dementia care for a maximum of 52 residents (median age 84 years; inter- quartile range (IQR): $76-89 ; 33 / 46$ female at the time of the second outbreak). Serological investigations in June 2020 found 33/66 (50.0\%) had SARS-CoV-2 anti- bodies after the first outbreak (18/32 residents; 15/34 staff).}

Care home $L$ provides residential and nursing care for a maximum of 64 residents (median age 85 years; IQR: $78-89 ; 36 / 57$ female at the time of the second outbreak). Serological investigation in May 2020 identified
59/117 (50.4\%) as seropositive (26/52 residents; $33 / 65$ staff).

\section{Laboratory investigations}

Nasal swabs were subjected to SARS-CoV-2 RT-PCR at the Public Health England (PHE) national reference laboratory as described previously [3]. Serological testing was conducted using in-house native virus lysate (PHE, UK) and receptor binding domain (RBD) EIA assays (PHE, UK), and a commercial nucleocapsid (N) assay (Abbott, Illinois, United States) [1,2,4]. Seropositivity was determined by reactivity in any assay; $>80 \%$ of samples were positive in $\geq 2$ assays. The native virus lysate assay was the most sensitive assay [4]. Neutralising antibody titres were determined by live virus neutralisation [2].

Whole genome sequencing was attempted on all RT-PCR-positive samples tested at the PHE reference laboratory as described previously [3]. Completed viral genomes were deposited in GISAID (Supplementary Table).

Protective effectiveness was estimated using two methods: risk ratios (RR) from a comparison of proportions (Fisher's exact test), and odd ratios (OR) from a penalised logistic regression model (Wald test).

A COVID-19 case was defined as any individual testing positive by RT-PCR for SARS-CoV-2, whether tested as a result of symptoms or through routine care home screening [5]. A re-infection was defined as an individual testing SARS-CoV-2 RT-PCR positive while having evidence of previous seropositivity by any assay, or a previous RT-PCR-positive result more than 90 days earlier in an individual without serological analysis (assumed to have seroconverted). 
Epidemic curves of outbreaks in care home A (A) and care home L (B), London, United Kingdom, September and October 2020

A. Epidemic curve of outbreaks in care home $A$

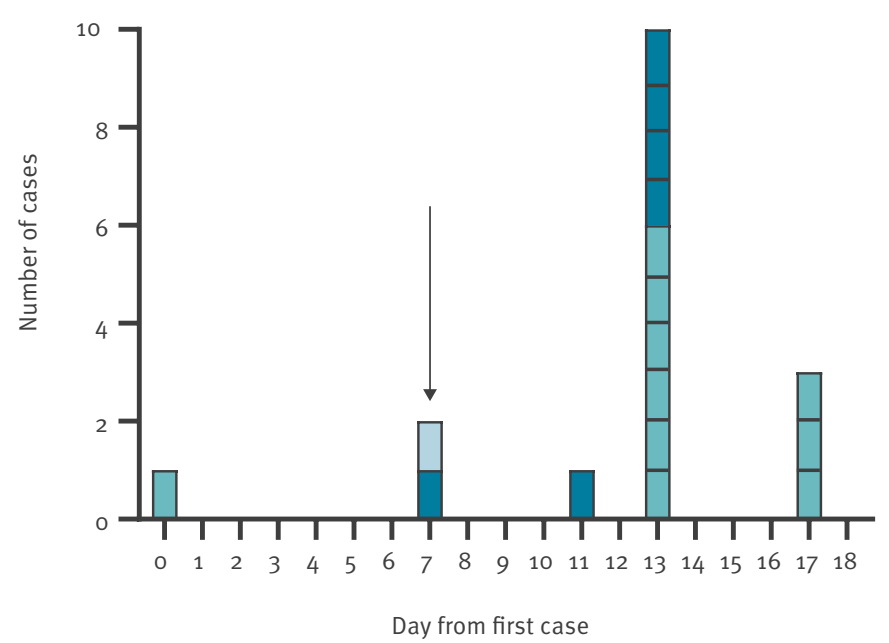

B. Epidemic curve of outbreaks in care home $L$

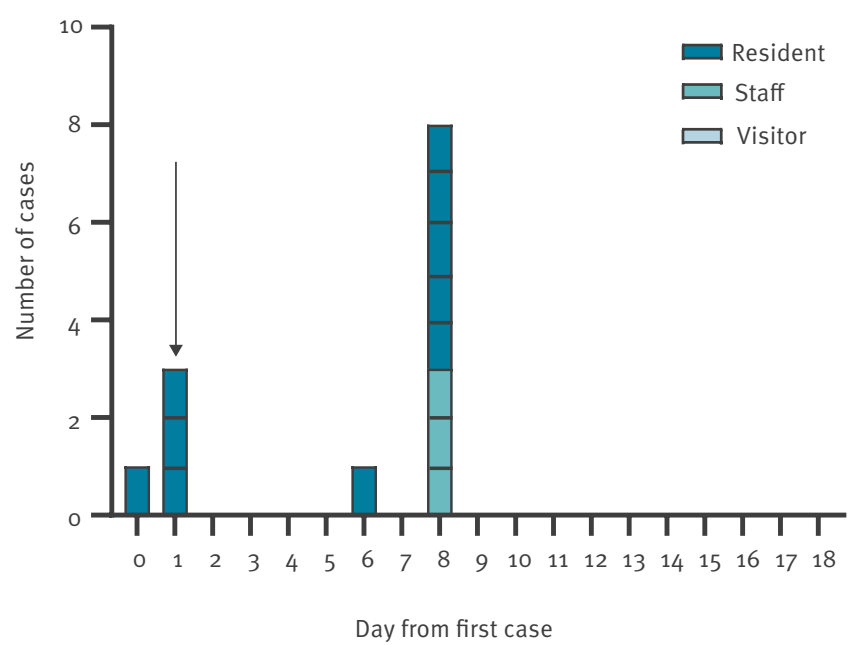

Mass testing occurred on day o and 7 as per national recommendations [5]. Clearance testing was conducted on day 28 , no new RT-PCR confirmed cases were identified in either care home on day 28 . These homes then returned to routine testing. Arrow indicates day outbreak declared.

\section{Ethical statement}

PHE has legal permission, provided by Regulation 3 of the Health Service (Control of Patient Information) Regulation 2002, to process patient confidential information for national surveillance of communicable diseases. The Investigation Protocol was reviewed and approved by the PHE Research Ethics and Governance Group (REGG) (Reference NRo204). Verbal consent for testing was obtained by care home managers from staff members and residents or their next of kin as appropriate.

\section{Outbreak evolution}

The outbreak in care home A began with a symptomatic staff member in mid-September 2020 (Figure $1 \mathrm{~A})$. Subsequent COVID-19 cases were identified in an asymptomatic visitor and asymptomatic resident on routine whole home screening 7 days later, prompting the declaration of an outbreak and instigating day o and 7 mass testing as per national recommendations, with clearance testing at day 28 prior to returning to routine screening patterns [5]. One further resident was identified following a swab taken for 'non-specific decline'. All other RT-PCR-positive individuals were identified through the mass outbreak screening conducted as feasible, depending on staff shifts, and all of them were asymptomatic throughout. Of 83 individuals (46 residents, 37 staff) that were swabbed,16 (6 residents, 10 staff) were RT-PCR positive, of whom two residents died, both within 1 week of testing positive. All but one of the COVID-19 cases were either seronegative $(n=7)$ or had unknown antibody status $(n=8)$ at the time of RT-PCR testing during the outbreak. The single previously seropositive staff member who was RT-PCR-positive is described below.
The outbreak in care home L began in October 2020. The index case was a resident identified on screening following hospital admission for a non-COVID-19 related condition and lethargy (Figure 1B). Following the identification, within a few days three asymptomatic residents were subsequently identified through routine whole home testing. Two of the residents went on to develop symptoms and the third became unresponsive and was admitted to hospital where they subsequently died 1 week after testing positive. One further resident was identified as positive following a swab taken for lethargy and weakness. All other RT-PCRpositive individuals were identified through whole care home outbreak screening. All staff identified as positive remained asymptomatic throughout. Residents identified as RT-PCR-positive exhibited a range of nonspecific symptoms including weakness, lethargy and loss of appetite. In total, 126 individuals (57 residents, 69 staff) were swabbed during the course of the mass outbreak screening; 13 (10 residents, 3 staff) were RT-PCR positive. All RT-PCR-positive residents and staff were either seronegative by all assays $(n=11)$ or had unknown antibody status $(n=2)$ at the time of RT-PCR testing.

There were no new COVID-19 cases identified in either care home on day 28 . These homes then returned to routine testing. The outbreaks were declared over at the end of October and beginning of November 2020 in homes $\mathrm{A}$ and $\mathrm{L}$, respectively.

\section{Genomics analysis}

The second COVID-19 outbreaks experienced by both care homes were due to SARS-CoV-2 strains that were genetically distinct from their respective first outbreaks 


\section{FIGURE 2}

Maximum likelihood phylogeny of SARS-CoV-2 genomes from individuals in care home A (panel A) and L (panel B) involved in the second outbreak in comparison to genomes from the first outbreak

A.

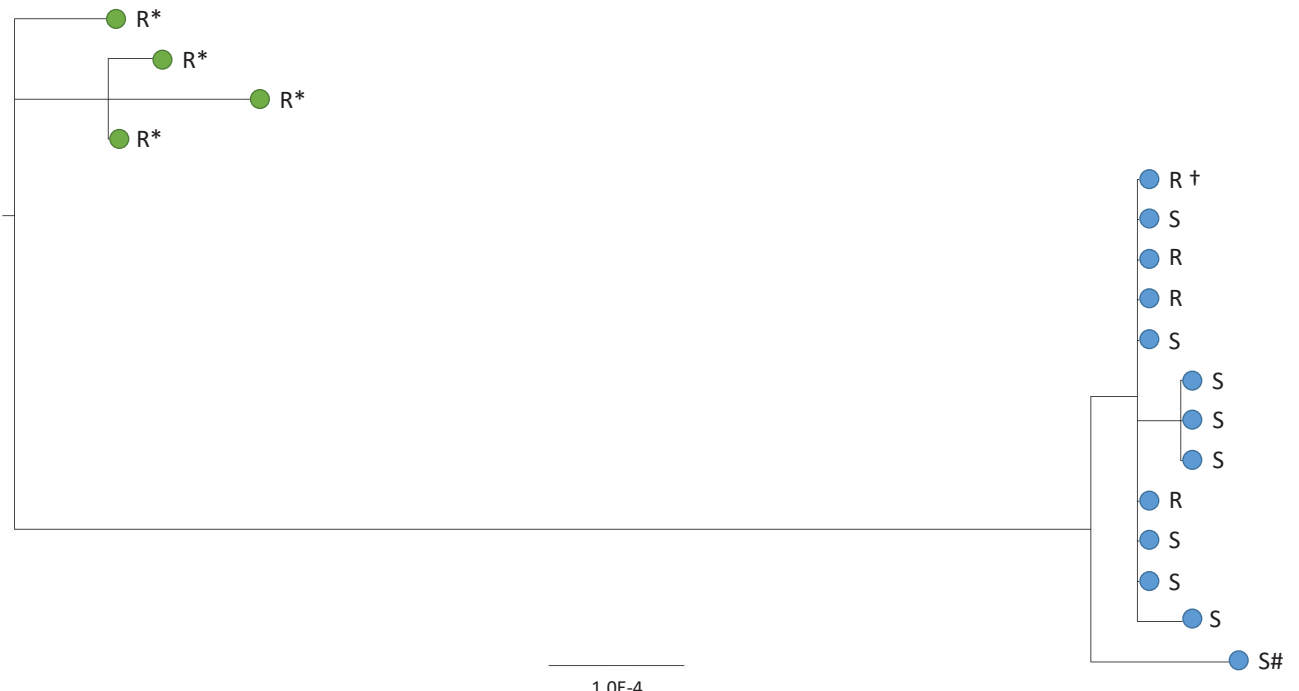

B.

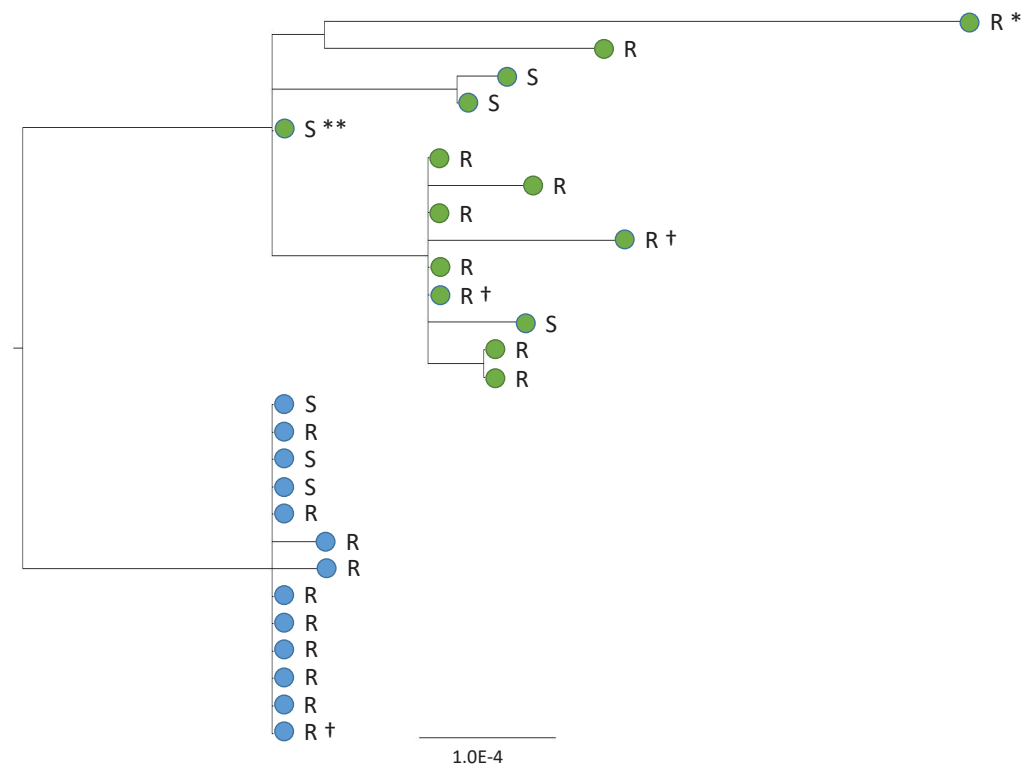

R: Resident; S: Staff member; $\uparrow:$ Fatality; *: S gene 614D variant; **: S gene 614D/G mixed base variant.

Unless otherwise specified all virus are 614G variant SARS-CoV-2 viruses.

Panel A: care home A, first outbreak in late April / early May 2020 (green dots) and second outbreak in late September / early October 2020 (blue dots).

Panel B: care home L, first outbreak in April 2020 (green dots) and second outbreak in October 2020 (blue dots).

Viral amplicons were sequenced using Illumina library preparation kits (Nextera kit, Illumina, Cambridge, UK) and sequenced on Illumina short-read sequencing machines (Nextseq or Hiseq sequencing platforms, Illumina, Cambridge, UK). The bioinformatics protocol to generate consensus sequences utilised Trimmomatic, BWA (mapping), and an in-house variant caller (quasibam) to align against a SARS $\mathrm{CoV}-2$ reference genome (NC 045512.2). Consensus sequences were generated using a depth cut-off of 20 reads and ambiguities called where a minority variant detected at $\geq 20 \%$, these were aligned using MAFFT (Multiple Alignment using Fast Fourier Transform, version 7.310), manually curated and maximum likelihood phylogenetic trees derived using IQtree (version 2.04). Genomes were included in analysis where the coverage of the reference genomes was $\geq 80 \%$. Completed viral genomes were deposited in GISAID (Supplementary Table). 
Severe acute respiratory syndrome coronavirus 2 attack rate by susceptibility status in two care home COVID-19 outbreaks, London, United Kingdom, September and October 2020

\begin{tabular}{|c|c|c|c|c|c|c|c|c|c|}
\hline \multirow[b]{2}{*}{ Category } & \multicolumn{3}{|c|}{ Residents $(n=103)$} & \multicolumn{3}{|c|}{ Staff $(n=106)$} & \multirow[b]{2}{*}{ Overall } & \multirow[b]{2}{*}{$\begin{array}{l}\text { Attack } \\
\text { rate }\end{array}$} & \multirow[b]{2}{*}{$\begin{array}{l}\text { Attack rate } \\
\text { percentage }\end{array}$} \\
\hline & $\begin{array}{c}\text { Status pre } \\
\text { outbreak }\end{array}$ & $\begin{array}{l}\text { PCR positive } \\
\text { during } \\
\text { outbreak }\end{array}$ & $\begin{array}{c}\text { PCR negative } \\
\text { during outbreak, } \\
\text { seroconversion }\end{array}$ & $\begin{array}{l}\text { Status } \\
\text { pre } \\
\text { outbreak }\end{array}$ & $\begin{array}{l}\text { PCR positive } \\
\text { during } \\
\text { outbreak }\end{array}$ & $\begin{array}{l}\text { PCR negative } \\
\text { during outbreak, } \\
\text { seroconversion }\end{array}$ & & & \\
\hline $\begin{array}{l}\text { Not } \\
\text { susceptible }\end{array}$ & $44^{a}$ & 0 & NA & 44 & 1 & NA & $88 / 209$ & $1 / 88$ & $1.1 \%$ \\
\hline Susceptible & 40 & 13 & 2 & 33 & 5 & 2 & $73 / 209$ & $22 / 73$ & $30.1 \%$ \\
\hline Unknown & 19 & 3 & NA & 29 & 7 & NA & $48 / 209$ & $10 / 48$ & $20.8 \%$ \\
\hline
\end{tabular}

COVID-19: coronavirus disease; NA: not applicable.

a Susceptibility of one case in this subset was determined by RT-PCR positivity and assumption of seroconversion as no serological result was available.

Susceptibility has been determined on basis of serological results before outbreaks using native viral antigen lysate EIA [2,4] or RBD [1] or Abbott Total N IgG, or the detection of RT-PCR positive without a corresponding serology. Two of the fatal cases were included in the susceptible cohort, whereas the third case had unknown susceptibility status.

which occurred during the first wave of the pandemic in spring 2020 (Figure 2). In care home A, virus strains from the earlier outbreak had $\mathrm{S}$ gene $614 \mathrm{D}$, whereas the strains in the later outbreak were 24-27 single nucleotide polymorphisms (SNPs) different and contained $\mathrm{S}$ gene $614 \mathrm{G}$. In the second outbreak, nine individuals were infected by an identical strain, which differed by 1-2 SNPs from three other COVID-19 cases. The individual with a probable re-infection (S\#) shared a virus sequence from B1.36 lineage and the same UK1350_1.2.1.1 phylotype as the other residents and staff, with 6 SNPs differences from the main cluster, including three mixed bases which were all outside the $S$ protein RBD coding region (Figure $2 \mathrm{~A}$ ). In care home $L$, virus strains from the earlier outbreak arose from several introductions and contained a mixture of $614 \mathrm{D}$ and $614 \mathrm{G}$ strains, whereas the second outbreak strains were all S gene $614 \mathrm{G}$ and differed by 11-18 SNPs from earlier strains (Figure 2B). In both care homes, fatal cases in residents had identical viral genomes to surviving residents.

\section{Serological analysis}

Based on serological investigations conducted after the first outbreaks in these care homes in April and May 2020, there were 73 seronegative individuals (40 residents, 33 staff) across the two care homes. Eighteen ( 13 residents, 5 staff) were RT-PCR positive during the second outbreaks, 16 (11 residents, 5 staff) of whom had blood taken after these outbreaks and all seroconverted in at least two assays. Additionally, four individuals (2 residents, 2 staff), who were both seronegative before the second outbreaks and SARS-CoV-2 RT-PCR negative during these outbreaks, subsequently seroconverted.

Only one seropositive staff member in care home A tested SARS-CoV-2 RT-PCR positive during the second outbreak investigation. This individual had been RT-PCR negative during the first outbreak in the spring and remained asymptomatic throughout. The staff member was subsequently SARS-CoV-2 antibody positive on all three serological assays in June 2020 but did not have detectable neutralising antibodies. Two weeks after the positive RT-PCR test at the second outbreak in the autumn, the individual had boosting of both $\mathrm{N}$ and $\mathrm{S}$ antibodies and developed neutralising antibodies to a titre of 1:1,057.

\section{Attack rate and protective effectiveness of previous exposure}

Only $1.1 \%(1 / 88)$ of individuals with confirmed previous SARS-CoV-2 exposure (antibody positive $(n=87)$ or RT-PCR positive $(n=1)$ ) became PCR-positive during the second outbreaks compared with $24.7 \%$ (18/73) of those with confirmed seronegative status before the second outbreaks (Table). Considering also the four previously seronegative individuals who tested RT-PCR negative during the second outbreak but had seroconverted after the outbreak, this gives a combined attack rate of $30.1 \%(22 / 73)$. The estimated RR was 0.038 (95\% Cl: $0.005-0.273$; $p<0.0001$ ) (Fisher's exact test) and the protective effectiveness estimate using RRs from a comparison of proportions [100*(1-RR)] was $96.2 \%$ (95\% Cl: $72.7 \%-99.5 \%)$. The estimated OR using a penalised logistic regression model was 0.039 (95\% Cl: $0.01-0.21 ; p<0.001$ ) (Wald test), with an effectiveness estimate $\left[100^{\star}(1-\mathrm{OR})\right]$ of $96.1 \%(95 \%$ Cl: $78.8 \%-99.3 \%)$.

\section{Discussion and conclusions}

Care homes have been disproportionately affected by the COVID-19 pandemic, with high rates of infection and deaths among the frail, elderly residents $[6,7]$. Nearly all survivors, however, develop high SARS-CoV-2 antibody levels, including neutralising antibodies, after infection $[2,8-10]$, but whether prior SARS-CoV-2 infection protects against re-infection is not known. In spring 2020, we investigated 13 London care homes, including the two care homes described here, and established prospective surveillance in this cohort of over 1,500 residents and staff. We identified high rates of SARS-CoV-2 infection among residents and staff during the first pandemic wave in the UK, most of whom were asymptomatic at the time of testing [3]. Follow-up serological assessments found that almost 
all RT-PCR-positive and two-thirds RT-PCR-negative residents and staff had SARS-CoV-2 antibodies, irrespective of age, sex or symptom status $[1,2]$.

In July 2020 , a national testing programme was implemented in care homes in the UK, with weekly swabbing for staff and 4-weekly swabbing for residents [11]. There were very few sporadic SARS-CoV-2 infections in these homes during the summer months but the two homes described here had second outbreaks during the autumn.

High rates of SARS-CoV-2 infection were detected in susceptible staff and residents during the second outbreaks in these two homes. Three of 16 susceptible SARS-CoV-2 RT-PCR-positive residents died. We found evidence of silent transmission, shown by seroconversion in RT-PCR-negative individuals, emphasising the continuing threat from SARS-CoV-2 in elderly people and the difficulty of maintaining control in closed settings. Prior infection with SARS-CoV-2 as determined by antibody or RT-PCR positivity was highly protective at 4 months. Only one re-infection occurred in a seropositive staff member, whose antibodies were boosted following re-infection. The first SARS-CoV-2 infection was not confirmed in this re-infection case, who was asymptomatic during both infections. This individual had $\mathrm{N}$ and $\mathrm{S}$ antibodies but did not have detectable neutralising antibodies following the first infection (data not shown), in keeping with other findings suggesting that functional antibody is an important correlate of protective immunity [12-14]. Taking all of these findings together highlights the importance comprehensive vaccination to protect vulnerable populations during the current pandemic.

Prior antibody testing with detailed public health and genomics investigations in the two care homes that experienced second COVID-19 outbreaks allowed the assessment of protection against re-infection, including protection against viruses with differences at position S 614, considered to be important for virus transmissibility [15].

\section{Acknowledgements}

The authors would like to thank the care home staff and residents across the 13 London care homes for their continued support of SARS-CoV-2 surveillance and outbreak investigations during the current pandemic.

\section{Conflict of interest}

None declared.

\section{Authors' contributions}

Study conception (SNL, JYC, MZ), study oversight (NI, JYC), oversight of laboratory work (MZ), protocol development (AJS, NI, SVW, JYC, SNL, MZ), data collection (AJS, NI, SVW, JYC, FA, JYC), operational conduct (AJS, NI, SVW, FA, MER, SNL, MZ), manuscript preparation (AJS, NI, SVW, JYC, MER,
SNL, MZ), PCR detection work (JE), serological assays (KH, $\mathrm{KB}, \mathrm{GA}, \mathrm{MP}, \mathrm{RG}$ ), laboratory genomics (SM, AL, SP), bioinformatics analyses (AL, SP) data analysis (AJS, NI, SVW, AC, SNL, MZ).

\section{References}

1. Jeffery-Smith A, Dun-Campbell K, Janarthanan R, Fok J, Crawley-Boevey E, Vusirikala A, et al. Infection and transmission of SARS-CoV-2 in care homes reporting no cases or outbreaks of COVID-19 in England: a prospective observational cohort study. Lancet Reg Heal Eur. 2021. Epub ahead of print. https://doi.org/10.1016/j.lanepe.2021.100038

2. Ladhani SN, Jeffery-Smith $A$, Patel M, Janarthanan R, Fok J, Crawley-Boevey E, et al. High prevalence of SARS-CoV-2 antibodies in care homes affected by COVID-19: Prospective cohort study, England. EClinicalMedicine. 2020;28:100597. https://doi.org/10.1016/j.eclinm.2020.100597

3. Ladhani SN, Chow IY, Janarthanan R, Fok J, Crawley-Boevey $\mathrm{E}$, Vusirikala A, et al. Investigation of SARS-CoV-2 outbreaks in six care homes in London, April 2020. EClinicalMedicine. 2020;26(0):100533. PMID: 32923993

4. Harvala H, Mehew J, Robb ML, ljaz S, Dicks S, Patel M, et al. Convalescent plasma treatment for SARS-CoV-2 infection: analysis of the first 436 donors in England, 22 April to 12 May 2020. Euro Surveill. 2020;25(28):2001260. https:// doi.org/10.2807/1560-7917.ES.2020.25.28.2001260 PMID: 32700670

5. UK Government. NHS. Care Home COVID-19 Testing Guidance. For testing of staff and residents. London: UK Government. NHS. Jan 2021. Available from: https://assets.publishing. service.gov.uk/government/uploads/system/uploads/ attachment_data/file/957849/Care_Home_Testing_Guidance_ England_v21-01.pdf

6. McMichael TM, Currie DW, Clark S, Pogosjans S, Kay M, Schwartz NG, et al. Epidemiology of covid-19 in a longterm care facility in King County, Washington. N Engl Med. 2020;382(21):2005-11. https://doi.org/10.1056/ NEJMoa2005412 PMID: 32220208

7. Patel MC, Chaisson LH, Borgetti S, Burdsall D, Chugh RK, Hoff CR, et al. Asymptomatic SARS-CoV-2 Infection and COVID-19 Mortality During an Outbreak Investigation in a Skilled Nursing Facility. Clin Infect Dis. 2020;71(11):2920-6. https://doi. org/10.1093/cid/ciaa763 PMID: 32548628

8. Wajnberg A, Mansour M, Leven E, Bouvier NM, Patel G, FirpoBetancourt $A$, et al. Humoral response and PCR positivity in patients with COVID-19 in the New York City region, USA: an observational study. Lancet Microbe. 2020;1(7):e2839. https://doi.org/10.1016/S2666-5247(20)30120-8 PMID: 33015652

9. Deeks JJ, Dinnes J, Takwoingi Y, Davenport C, Spijker R, TaylorPhillips S, et al. Antibody tests for identification of current and past infection with SARS-CoV-2. Cochrane Database Syst Rev. 2020;6(6):CD013652. https://doi.org/10.1002/14651858. CD013652 PMID: 32584464

10. Graham NSN, Junghans C, McLaren R, Randell P, Lang N, Ladhani SN, et al. High rates of SARS-CoV-2 seropositivity in nursing home residents. J Infect. So163-4453(20)30574-0. 27 Aug. 2020, https://doi.org/10.1016/j.jinf.2020.08.040

11. Ladhani SN, Chow JY, Atkin S, Brown KE, Ramsay ME, Randell P, et al. Regular mass screening for SARS-CoV-2 infection in care homes already affected by COVID-19 outbreaks: Implications of false positive test results. J Infect. 2020;0(0):4840. https:// doi.org/10.1016/j.jinf.2020.09.008 PMID: 32949618

12. Addetia A, Crawford KHD, Dingens A, Zhu H, Roychoudhury P, Huang ML, et al. Neutralizing Antibodies Correlate with Protection from SARS-CoV-2 in Humans during a Fishery Vessel Outbreak with a High Attack Rate. J Clin Microbiol. 2020;58(11):e02107-20. https://doi.org/10.1128/JCM.02107-20 PMID: 32826322

13. Yu J, Tostanoski LH, Peter L, Mercado NB, McMahan K, Mahrokhian SH, et al. DNA vaccine protection against SARSCoV-2 in rhesus macaques. Science, 2020;369(6505):806-11. https://doi.org/10.1126/science.abc6284 PMID: 32434945

14. Lumley SF, O'Donnell D, Stoesser NE, Matthews PC, Howarth A, Hatch SB, et al. Antibody Status and Incidence of SARS CoV-2 Infection in Health Care Workers. N Engl J Med. 2020 Dec 23:NEJMoa2034545. https://doi.org/10.1056/NEJMoa2034545. Epub ahead of print. PMID: 33369366; PMCID: PMC7781098.

15. Korber B, Fischer WM, Gnanakaran S, Yoon H, Theiler J, Abfalterer W, et al. Tracking Changes in SARS-CoV-2 Spike: Evidence that D614G Increases Infectivity of the COVID-19 Virus. Cell. 2020;182(4):812-827.e19. https://doi. org/10.1016/j.cell.2020.06.043 PMID: 32697968 
License, supplementary material and copyright

This is an open-access article distributed under the terms of the Creative Commons Attribution (CC BY 4.0) Licence. You may share and adapt the material, but must give appropriate credit to the source, provide a link to the licence and indicate if changes were made.

Any supplementary material referenced in the article can be found in the online version.

This article is copyright of the authors or their affiliated institutions, 2021. 